\title{
SOME ADVANCES IN THE ANALYSIS OF SEMIDEFORMABLE MEDIA
}

\author{
Theodore Sussman† and Klaus-JUrgen Bathe $\ddagger$ \\ †ADINA R \& D Inc., 71 Elton Avenue, Watertown, MA 02172, U.S.A. \\ †Department of Mechanical Engineering, Massachusetts Institute of Technology, Cambridge, MA \\ 02139, U.S.A. \\ Abotract-Semideformable media are materials in which certain modes of deformation are prohibited \\ (for example, incompressible materials). The displacement/pressure $(u / p)$ finite element formulation can \\ be used to effectively analyze semideformable media. We summarize the basic characteristics of the \\ formulation and present some representative numerical examples in linear and nonlinear analysis.
}

\section{INTRODUCTION}

The displacement-based finite element method, although it can be used for a wide variety of problems, is inefficient when analyzing materials that are almost semideformable. A material is semideformable when, due to its constitutive properties, certain modes of deformation are prohibited. An important class of semideformable materials are incompressible materials, although, as discussed below, not all semideformable materials are incompressible.

Much research effort over the last 20 years has been devoted to the development of the finite element method for the analysis of incompressible materials [1-6]. One fruitful concept employed is the separate interpolation of a stress variable related to the pressure in addition to the usual displacement interpolations. Many formulations based on this concept have been developed, but frequently these formulations are either quite specialized (for example, formulations developed only for rubber-like materials), only valid for almost incompressible analysis or require the use of specialized solution techniques when solving the finite element system of equations.

In this paper, we summarize a formulation that we recently developed that is widely applicable in solutions involving elastic and inelastic materials that are compressible, almost incompressible or otherwise almost semideformable. This formulation, called the $u / p$ formulation, is discussed in detail in [7]. Characteristics of this formulation include a single derivation for a wide range of materials and kinematic assumptions and the use of quantities with clear physical meanings. The examples presented in this paper show the use of the $u / p$ formulation in orthotropic analysis and in the analysis of rubber components.

\section{SUMMARY OF THEORY}

The name of our formulation, the $u / p$ formulation, reflects the use of separate interpolations for the displacements and the (hydrostatic) pressure. The basic concept underlying the $u / p$ formulation is the inclusion of separate pressure degrees-of-freedom in a manner almost independent of the material description. To achieve this, we state three reasonable requirements that the formulation should satisfy and then, after presenting the formulation, show that it satisfies the requirements.

The requirements are:

(1) The tangent stiffness matrix corresponding to the incremental displacements and pressures should be symmetric.

(2) The pressure computed from the stress tensor should equal the separately interpolated pressure.

(3) The constraint equation relating the separately interpolated pressure to the displacement field should incorporate, in an average sense, the pressure-strain material relationship.

The total Lagrangian version of our formulation is

$$
\delta\left(\int_{0_{Y}}\left({ }_{0} \tilde{W}-\frac{1}{2_{0} P(\tilde{p})}\left({ }^{\prime} \bar{p}-{ }^{\prime} \tilde{p}\right)^{2}\right){ }^{\circ} \mathrm{d} V\right)=\mathscr{R},
$$

where

$$
\begin{aligned}
& { }_{0}^{\prime} W=\text { the material potential }\left(d_{0}^{\prime} W={ }_{0}^{\prime} \bar{S}_{4} d_{0}^{\prime} \varepsilon_{i j}\right) \\
& { }^{\prime} \bar{p}=\text { the pressure as computed from the displace- } \\
& \text { ment field } \\
& { }_{0}^{\prime} \tilde{p}=\text { the separately interpolated pressure } \\
& { }_{0} P(\cdot)=\text { the operation that extracts the pressure } \\
& \text { from the material potential }
\end{aligned}
$$




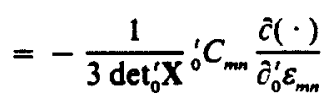

and other symbols are those used in [8]. This formulation can be used for material descriptions that satisfy

$$
{ }_{0} P(' \bar{p})=\text { constant. }
$$

Notice that the $u / p$ formulation is stated in potential form [i.e. the variation of the internal potential equals the variation of the external (loads) potential]. This implies that the tangent stiffness matrix is symmetric.

The allowed variations are those with respect to displacement degrees-of-freedom and those with respect to pressure degrees-of-freedom. Taking variations of eqn (1) with respect to displacements gives

$$
\int_{0 V}\left[{ }_{0} \mathcal{S}_{k 1}-\frac{1}{{ }_{0} P\left({ }^{\prime} \bar{p}\right)}\left({ }^{\prime} \bar{p}-' \tilde{p}\right) \frac{\partial^{\prime} \bar{p}}{\partial_{0}^{\prime} \varepsilon_{k 1}}\right] \delta_{0}^{\prime} \varepsilon_{k 1}{ }^{0} \mathrm{~d} V=\mathscr{R} .
$$

We can identify the second Piola-Kirchhoff stress tensor as the quantity in brackets in eqn (3). Using standard transformations and the definition of ${ }_{0} P\left({ }^{\prime} \bar{p}\right)$ given above,

$$
\begin{aligned}
& ' p=-\frac{1}{3} \tau_{m m}
\end{aligned}
$$

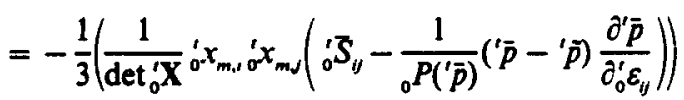

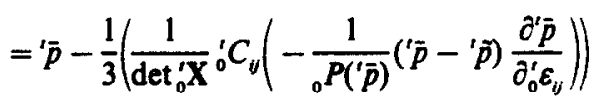

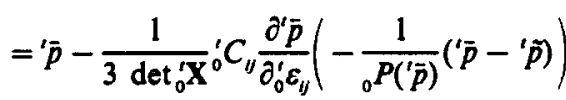

$$
\begin{aligned}
& ={ }^{\prime} \bar{p}-\left({ }^{\prime} \bar{p}-' \tilde{p}\right)=' \tilde{p}
\end{aligned}
$$

which shows that requirement (2) is satisfied.

Taking variations of eqn (1) with respect to the separately interpolated pressure gives

$$
\int_{\operatorname{lo} 0} \frac{1}{P\left({ }^{\prime} \bar{p}\right)}\left({ }^{\prime} \bar{p}-' \bar{p}\right) \delta \tilde{p}^{\circ} \mathrm{d} V=0
$$

which is satisfied when ' $\tilde{p}$ equals ' $\bar{p}$ in an average sense over each element (because we later choose pressure interpolations that are element-based). Since ' $\bar{p}$ is evaluated from the pressure-strain material relationship, requirement (3) is satisfied.

We observe that if ' $\tilde{p}$ equals ' $\bar{p}$ everywhere, the $u / p$ formulation reduces to the displacement-based formulation. One consequence is that the stiffiness matrix and force vector from the $u / p$ formulation are identical to the results from the displacement- based formulation when the number of pressure variables in each element equals the number of integration points in the element.

To derive the finite element force vector and stiffness matrix, it is necessary to employ chain differentiation. The results may be placed in the form

$$
\left[\begin{array}{ll}
\mathbf{K U U} & \mathbf{K U P} \\
\mathbf{K} \mathbf{X P}^{T} & \mathbf{K P P}
\end{array}\right]\left[\begin{array}{l}
\hat{\mathbf{u}} \\
\hat{\mathbf{P}}
\end{array}\right]=\left[\begin{array}{c}
t^{+}{ }^{\prime} \mathbf{R} \\
\mathbf{0}
\end{array}\right]-\left[\begin{array}{l}
\mathbf{F U U}^{\mathbf{F U}} \\
{ }^{\mathbf{F P}}
\end{array}\right],
$$

where

'KUU, KUP, 'KPP = partitions of the tangent stiffness matrix

'FU $=$ vector corresponding to element stresses

'FP = out-of-balance pressure constraints vector

i $+{ }^{\Delta} \mathbf{R}=$ external loads vector

$\mathbf{Q}=$ increments in displacement variables

$\hat{p}=$ increments in pressure variables

and the entries of these matrices are given in [7]. As in displacement-based finite element analysis, an equilibrium solution is one in which the right-handside of eqn (5) is identically zero.

We could solve the equations in the above form. However, because we use pressure discretizations that are discontinuous between elements, it is more efficient to statically condense out the pressure degrees-of-freedom at the element level. This process yields an element stiffness matrix and force vector involving only displacement degrees-of-freedom, and these matrices are then assembled into the global stiffness matrix and force vector in the usual way.

We note that in contact analysis, we use the outof-balance force vector $\mathbf{R}-$ 'FU in calculating reactions, not the out-of-balance force vector produced from the static condensation procedure.

An important consideration when using the $u / p$ formulation is the selection of the number of pressure degrees-of-freedom per element. We consider, as mentioned above, pressure interpolations that are element-based, i.e. for two-dimensional elements,

$$
{ }^{\prime} \tilde{p}={ }^{\prime} p_{1}+{ }^{\prime} p_{2} r+{ }^{\prime} p_{3} s+{ }^{\prime} p_{4} r s+\ldots,
$$

where ${ }^{\prime} p_{1}, p_{2} \ldots$ are internal element variables and $r, s$ are the isoparametric coordinates. Much research has been conducted to determine the best number of pressure variables for similar formulations [9-11] and, based on the Brezzi-Babuska condition, a conservative approach is to use a nine-node isoparametric element with three pressure variables in two-dimensional analysis and to use a 27 -node isoparametric element with four pressure variables in three-dimensional analysis. These elements are abbreviated below as $9 / 3$ and $27 / 4$ elements, respectively. 


\section{NUMERICAL EXAMPLES}

We now present some sample analyses. These analyses were run using the ADINA system [12]. Unless otherwise specified, the following solution procedures are employed. A $3 \times 3$ Gaussian integration is used to integrate the element stiffness matrices and force vectors in two-dimensional analysis and $3 \times 3 \times 3$ Gaussian integration is used in threedimensional analysis. A simple load stepping algorithm is employed; for each load step, the prespecified load (or displacement) increment is applied and full Newton equilibrium iterations are used to reestablish equilibrium. Note that no special procedures, such as those described in [4] and [5], are employed.

3.1 Orthotropic linear elasticity analysis of a cylinder under internal pressure

As an example analysis in which the $u / p$ formulation is effectively used for a semideformable material that is not incompressible, we consider the axisymmetric analysis of an orthotropic cylinder under internal pressure loading (Fig. 1). The material constants for the cylinder are

$$
\begin{aligned}
& E_{r}=10^{6} \mathrm{~g} \mathrm{MPa}, E_{\theta}=10^{6} \mathrm{MPa}, E_{z}=10^{6} \mathrm{MPa} \\
& v_{r \theta}=\frac{0.25}{g}, v_{\theta z}=0.25, v_{r z}=\frac{0.25}{g} \\
& G_{r z}=4 \times 10^{5} \mathrm{MPa},
\end{aligned}
$$

where $r, \theta, z$ denote the coordinate axes and $g$ is a number used to control the degree of orthotropy. The pressure-strain relationship for this material is [7]

$$
\begin{aligned}
' \bar{p}= & -\frac{1}{3} \frac{10^{6} g}{15 g-5 / 2}\left[(15 g+10)^{\prime} e_{m}\right. \\
& \left.+25^{\prime} e_{\theta \theta}+25^{\prime} e_{z z}\right]
\end{aligned}
$$

and we note that when $g=1 / 6$, the pressure becomes decoupled from the strains; i.e. the material must deform so as to satisfy the requirement

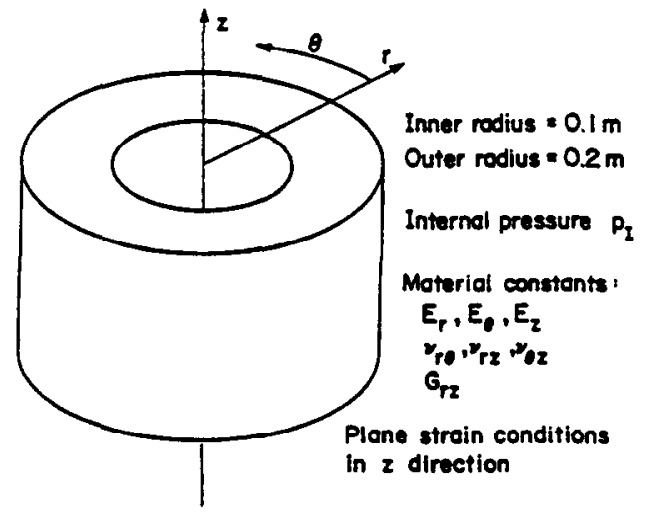

Fig. 1. Orthotropic cylinder under internal pressure.

$$
\frac{1}{2} e_{r r}+e_{\theta \theta}+e_{s z}=0 .
$$

Equation (7) shows that the material is semideformable.

Our four element finite element mesh is shown in Figure 2. Table 1 shows the root-mean-square pressure, defined by

$$
P_{\mathrm{rms}}=\sqrt{\frac{\int_{v} p^{2} \mathrm{~d} V}{\int_{v} \mathrm{~d} V}}
$$

for different values of $g$ when nine-node displacementbased elements and $9 / 3 u / p$ formulation elements are used. The displacement-based element mesh locks, with the root-mean-square pressure increasing by a factor of 260 , whereas the $u / p$ element mesh does not lock. Figure 3 shows stress graphs from the $9 / 3$ element mesh solution for $g=0.16666667$ and it is seen that the solution is quite accurate.

\subsection{Analysis of rubber components}

We use the following form of the Mooney-Rivlin material description when analyzing rubber-like materials:

$$
\begin{aligned}
: \bar{W}= & C_{1}\left({ }_{0}^{\prime} I_{1}^{\prime} I_{3}^{\prime} I^{-1,3}-3\right)+C_{2}\left({ }_{0}^{\prime} I_{2} o_{0}^{\prime} I_{3}^{-2 / 3}-3\right) \\
& +\frac{1}{2} \kappa\left({ }_{0}^{0} I_{3}^{1 / 2}-1\right)^{2},
\end{aligned}
$$

where $C_{1}, C_{2}$ are the Mooney-Rivlin material constants, ${ }_{0}^{\prime} I_{1},{ }_{0}^{1} I_{2},{ }_{0}^{1} I_{3}$ are the invariants of the Cauchy-Green deformation tensor and $\boldsymbol{x}$ is a bulk modulus. The factors of ${ }_{0}^{1} I_{3}^{-1 / 3},{ }_{0}^{1} I_{3}^{-2 / 3}$ that appear are necessary to make the pressure-strain relationship simple, i.e.

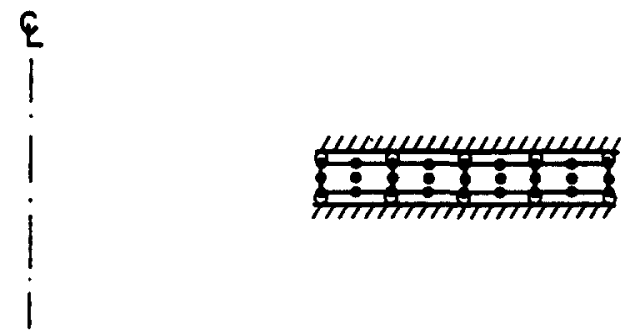

Fig. 2. Four $9 / 3$ element mesh for orthotropic cylinder.

Table 1. Root-mean-square pressure vs $g$ for orthotropic cylinder problem

\begin{tabular}{lcc}
\hline \multicolumn{1}{c}{$g$} & $\begin{array}{c}P_{\text {rms }} \\
\text { (disp-based) }\end{array}$ & $\begin{array}{c}P_{\text {rmas }} \\
(u / p)\end{array}$ \\
\hline 1 & 27.78 & 27.78 \\
0.5 & 25.53 & 25.51 \\
0.2 & 21.28 & 19.38 \\
0.17 & 85.39 & 17.77 \\
0.167 & 754.7 & 17.59 \\
0.1667 & 3919 & 17.57 \\
0.16667 & 6752 & 17.56 \\
0.166667 & 7281 & 17.57 \\
0.1666667 & 7338 & 17.57 \\
0.16666667 & 7343 & 17.57 \\
\hline
\end{tabular}



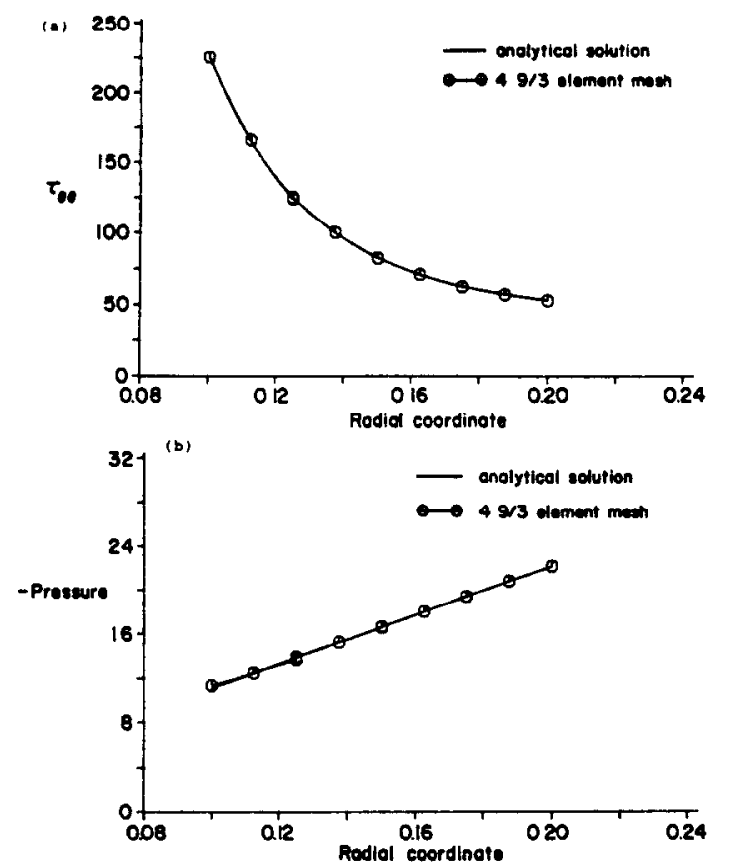

Fig. 3. Stress line graphs for orthotropic cylinder. (a) Hoop stress. (b) Pressure.

$$
\bar{p}=-\kappa\left({ }_{0}^{\prime} I_{3}^{\prime 2}-1\right) .
$$

We regard the bulk modulus not as a penalty parameter but rather as a material property of the rubber. Hence we model rubber as a slightly compressible material, an assumption with some experimental justification.

A similar approach may be used to derive an Ogden material relationship with a bulk modulus [7].

3.2.1. Analysis of a rubber ring. A problem of practical engineering importance is the determination of stresses within a toroidal rubber ring that is pressed between two plates. In our previous paper, we employed a plane strain idealization; however, on further thought, we decided that an axisymmetric idealization as shown in Fig. 4 is more appropriate. Two cases are considered here, frictionless contact and frictional contact with a Coulomb coefficient of 0.7 .

An approximate force-deflection relationship based on experimental data is given by Lindley as [13]

$$
\frac{R}{\pi d D E}=1.25\left(\frac{\Delta}{d}\right)^{32}+50\left(\frac{\Delta}{d}\right)^{6},
$$

where $R$ is the applied load, $D$ is the mean ring diameter, $d$ is the diameter of the rubber crosssection, $E$ is the Young's modulus (assumed constant) and $\Delta$ is the applied displacement. This equation fits experimental data obtained from rubber rings vulcanized from a gum compound.

An approximate analytical solution based on a plane strain idealization and the compression modulus concept is given by [14]

$$
\frac{R}{\pi d D E}=\alpha+\kappa \beta
$$

where

$$
\begin{aligned}
\alpha & =\frac{4}{3}\left[\ln \left(\tan \left(\frac{\theta}{2}+\frac{\pi}{4}\right)\right)-\sin \theta\right] \\
\beta & =\frac{1}{3}\left(\frac{\sin ^{3} \theta}{2 \cos ^{2} \theta}-\frac{9}{8} a\right) \\
\cos \theta & =1-\frac{\Delta}{d}
\end{aligned}
$$

$k=$ empirical factor less than unity, used in computing the compression modulus.

We idealize the rubber using a Mooney-Rivlin description with constants given in Fig. 4. The finite element mesh employed is shown in Fig. 5 and includes 54 9/3 elements. Contact surfaces (shown with thick lines) are used to model the contact conditions.

In the finite element analyses, we apply a total displacement of $0.3 \mathrm{~cm}$ in 10 equal load steps. The resulting force-deflection curves are shown in Fig. 6 and the deformed meshes are shown in Fig. 7. We observe that the friction prevents expansion of the ring in the radial direction and hence, when friction is included, the assumption of plane strain deformation is valid. Observing the force-deffection curves, we see that the addition of friction stiffens the ring, as expected. The two comparison solutions are both softer than the finite element solution with friction and these differences are probably due to the approximations made in deriving eqn (12) and also to our choice of material relationship.

Figure 8 shows the pressures within the ring for a deflection of $0.3 \mathrm{~cm}$. The effect of friction is to increase the pressure for a given deformation. The pressure bands are continuous, indicating that the mesh is sufficiently refined [15]. Notice that the magnitude of the pressure is on the order of the material shear modulus.

3.2.2 Torsion of a rubber cylinder. The solid rubber cylinder shown in Fig. 9 is constrained in the axial direction and is twisted by the applied moment. This problem was solved analytically by Rivlin, assuming total incompressibility. The solutions are [16]

$$
\begin{aligned}
& M=\pi\left(C_{1}+C_{2}\right) r^{4}\left(\frac{\theta}{L}\right) \\
& N=\frac{\pi}{2}\left(C_{1}+2 C_{2}\right) r^{4}\left(\frac{\theta}{L}\right)^{2},
\end{aligned}
$$

where $M$ is the applied moment, $N$ is the axial force (positive in compression), $r$ is the cylinder radius, $L$ is the cylinder length and $\theta$ is the total angular 


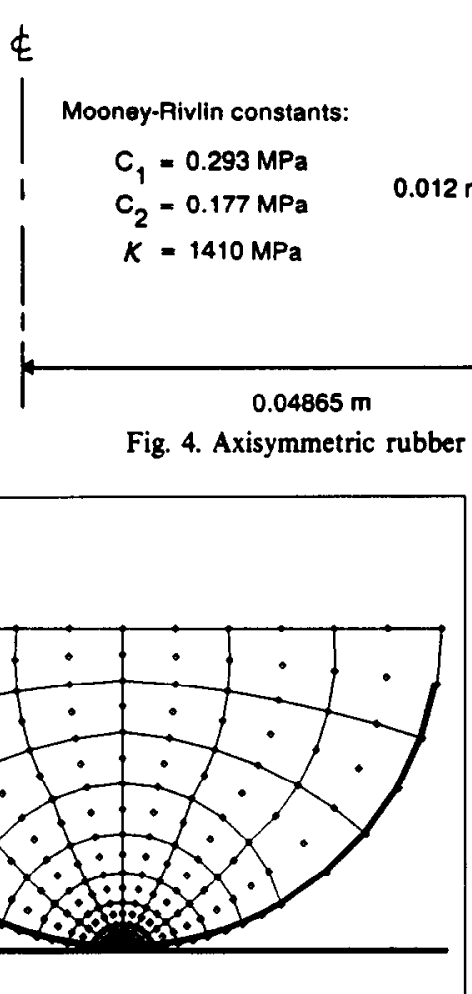

Fig. 5. Fifty-four $9 / 3$ element mesh for rubber ring (thick lines are contact surfaces).

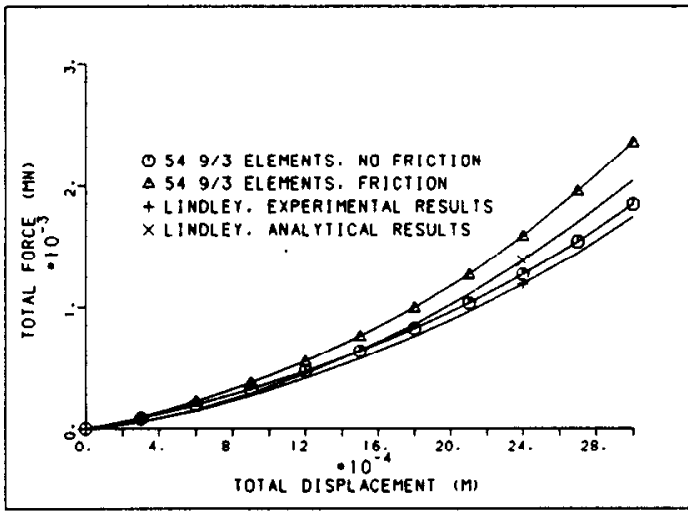

Fig. 6. Force-deflection curves for rubber ring.

displacement. (In this problem, the cylinder radius does not change as the cylinder is twisted because of the incompressibility constraint.)

We use the mesh layout of eight collapsed 27 . node elements shown in Fig. 10 to model the cylinder. On the far end of the cylinder, all of the nodes are fixed. On the near end, the angular displacement of the center node is prescribed and rigid links placed between this node and the other nodes on the near end cause the entire cross-section to rotate by this amount. A stiff truss element is connected to the center node and ground; this element constrains the cylinder in the axial direction and is used to measure the axial reaction force.

We apply a total angular displacement of 0.1 radians in 10 equal load steps in two analyses, one using displacement-based elements and the other
Prescribed displacement

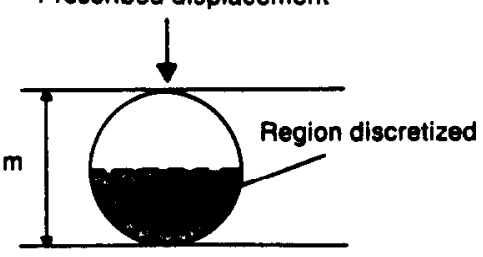

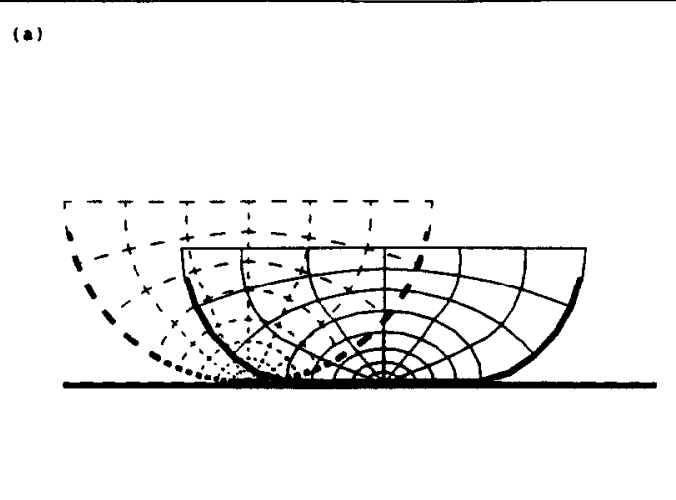

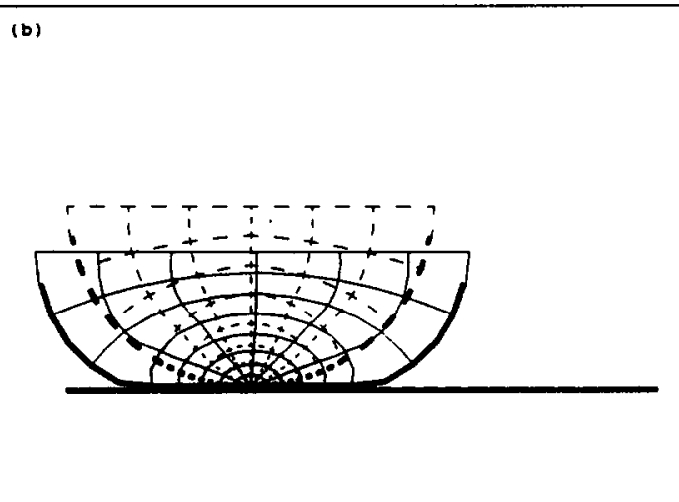

Fig. 7. Deformations of rubber ring for $\Delta=0.3 \mathrm{~cm}$. (a) $\mu=0.0$. (b) $\mu=0.7$.

using $u / p$ elements each with four pressure degrees of freedom. The moment-rotation curves are shown in Fig. 11 where we see that the two finite element solutions agree very well with the analytical solution. The axial force-rotation curves are shown in Fig. 12. Here the $u / p$ element solution agrees with the analytical solution but the displacement-based element solution stiffens excessively as the rotation is increased. Notice that the displacements of the mesh are still quite small at a rotation of 0.1 radians (Fig. 13).

3.2.3 Analysis of a conical rubber spring. As an illustration of some of the difficulties associated with rubber analysis, we present the partial analysis of the conical rubber spring shown in Fig. 14. The goal of the analysis is to determine the force-deflection curve and the stress state at various deformations. 

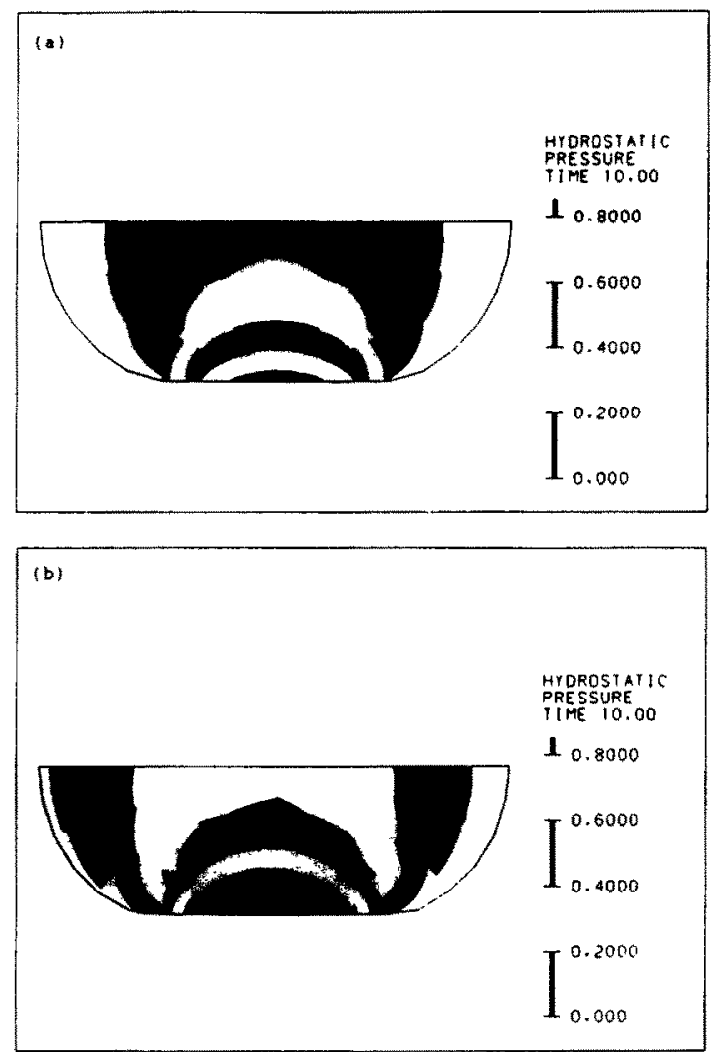

Fig. 8. Pressure band plots of rubber ring for $\Delta=0.3 \mathrm{~cm}$ (plots may also be produced in color for easier visualization). (a) $\mu=0.0$. (b) $\mu=0.7$.

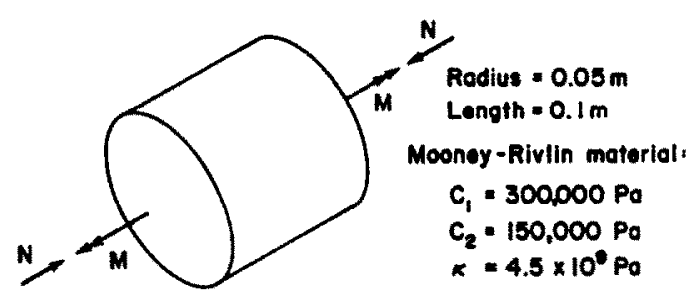

Cylinder constroined to heve no diepiacements in the oxlol direction.

Fig. 9. Torsion of a rubber cylinder.

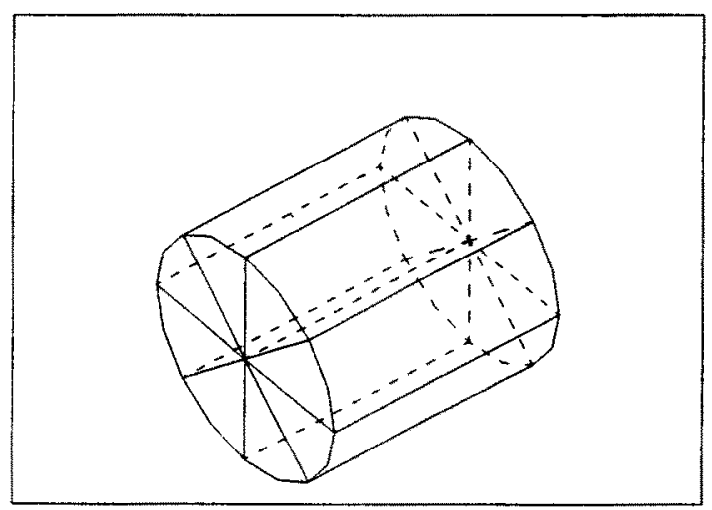

Fig. 10. Eight 27-node-element mesh for rubber cylinder.

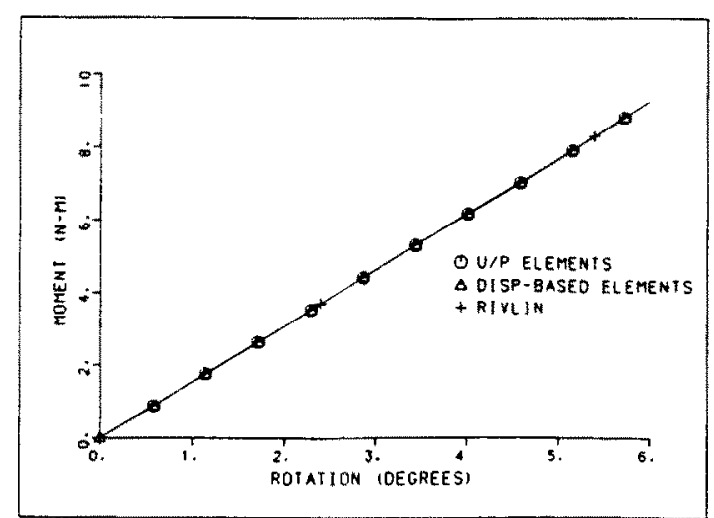

Fig. 11. Moment-rotation curves for rubber cylinder.

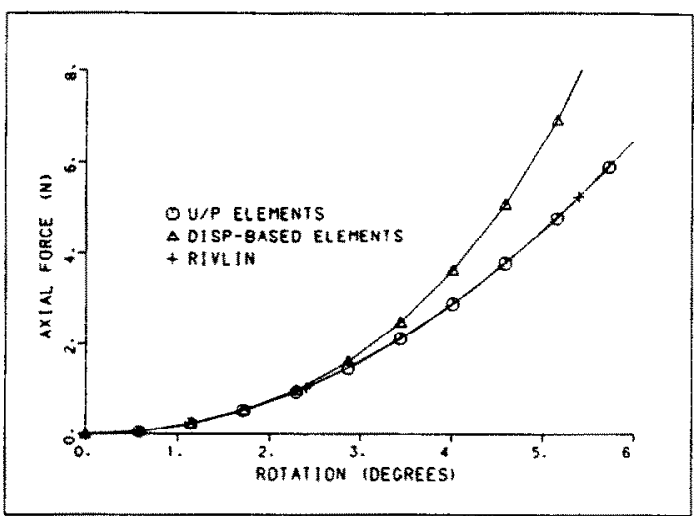

Fig. 12. Axial force-rotation curves for rubber cylinder.

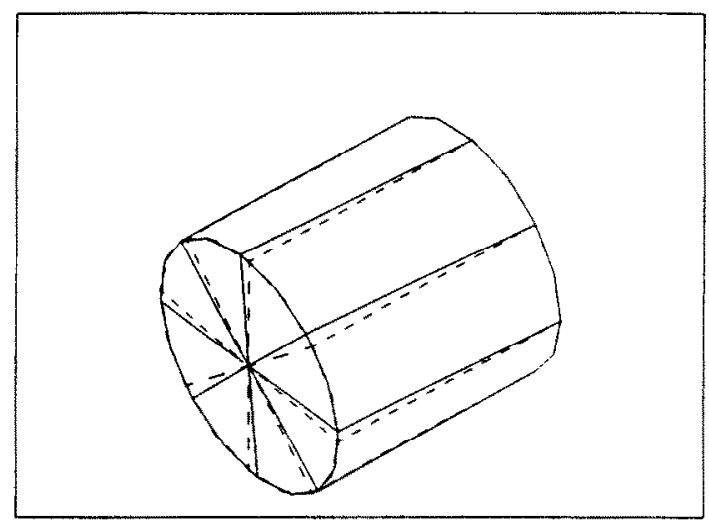

Fig. 13. Deformations of rubber cylinder for $\theta=0.1$ radians.

This problem was previously considered by Zdunek and Bercovier [6]. The geometric data was obtained from Zdunek as the nodal point locations of the 67 9/3 element mesh shown in Fig. 15.

We employ simple load stepping with the $679 / 3$ element mesh and a $674 / 1$ element mesh (four-node elements with constant pressures). Figure 16 shows the force-deflection curves. The analyses basically agree up to a displacement of $3.87 \mathrm{~mm}$ and the 67 $4 / 1$ element mesh results are comparable to those presented by Zdunek and Bercovier. At $3.87 \mathrm{~mm}$, we find a zero eigenvalue in the tangent stiffness matrix for the $679 / 3$ element mesh and the program solution stops. Figure 17 shows the undeformed and deformed $679 / 3$ element meshes for $\Delta=3.87 \mathrm{~mm}$ and we notice that a crease has formed on the upper 
(a)
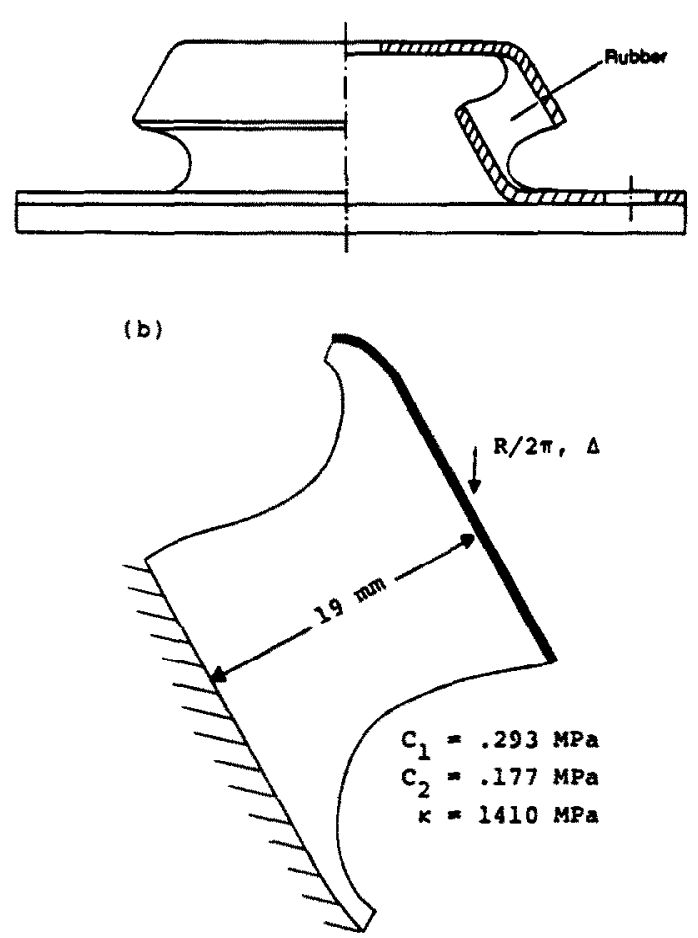

Fig. 14. Rubber spring geometry. (a) Schematic drawing. (b) Spring cross-section.

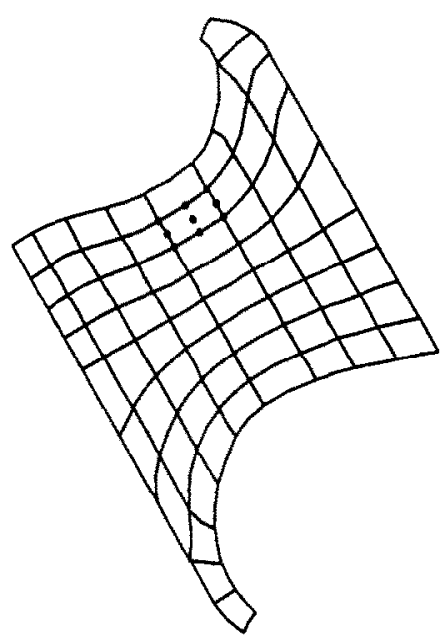

Fig. 15. Sixty-seven $9 / 3$ element mesh for rubber spring. surface of the spring. This crease is the source of the difficulty; the eigenvector associated with the zero eigenvalue at $\Delta=3.87 \mathrm{~mm}$ represents an instability at the crease (Fig. 18). We also note that the largest pressure in the creased element is larger than the material shear modulus.

Further analyses using refined meshes, employing both $9 / 3$ elements and $4 / 1$ elements, all exhibited the same difficulty. We hypothesize that a very fine mesh (perhaps using rezoning) and the use of a load-displacement constraint algorithm [12] may be required to properly model the creasing. A very coarse mesh, such as the $674 / 1$ element mesh, does

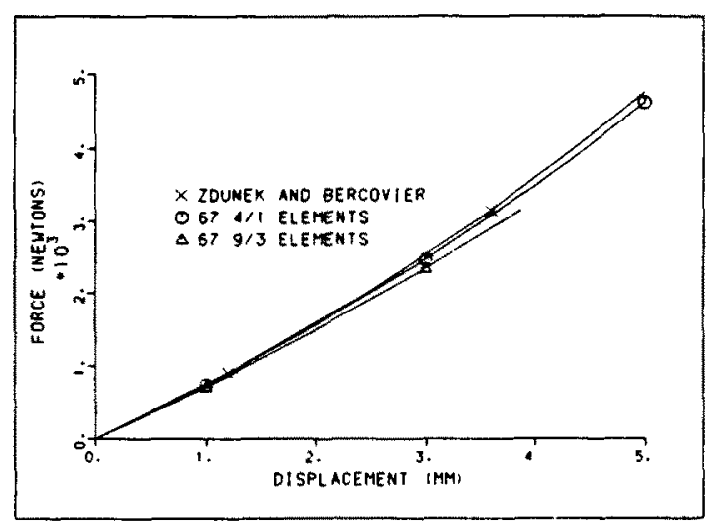

Fig. 16. Force-deflection curves for rubber spring (locations of symbols do not necessarily correspond to solution steps).

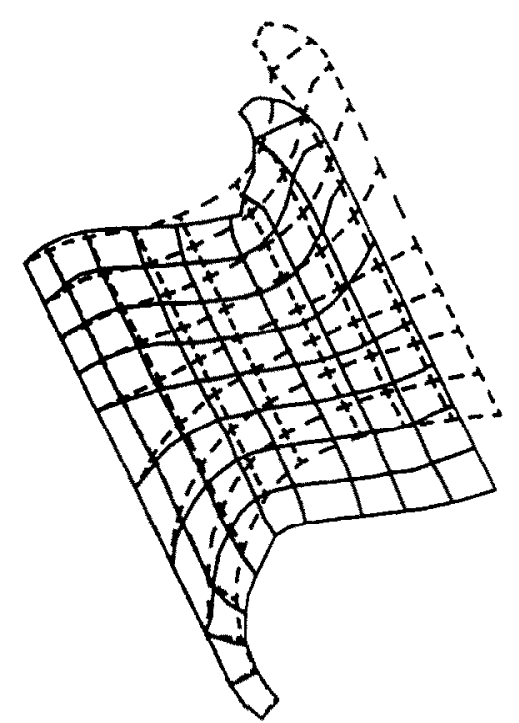

Fig. 17. Deformations of rubber spring, $679 / 3$ element mesh, $\Delta=3.87 \mathrm{~mm}$.

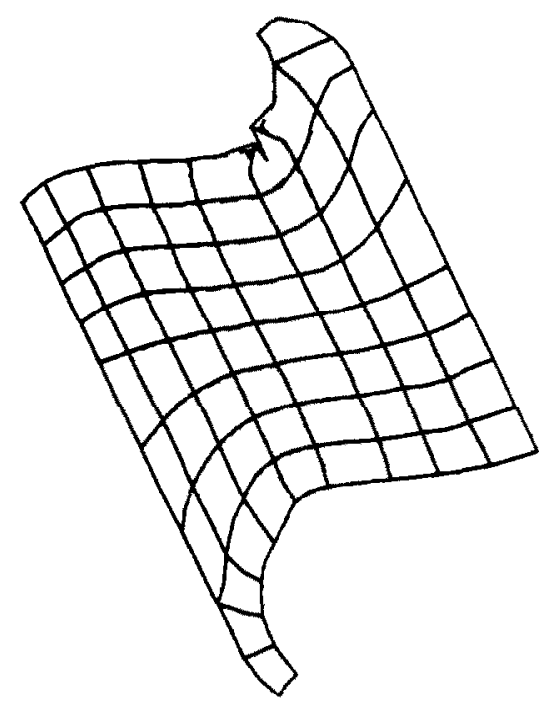

Fig. 18. Eigenvector corresponding to the zero eigenvalue of the tangent stifness matrix, 679.3 element mesh, $\Delta=3.87 \mathrm{~mm}$. 
not model the crease at all and this is why solution difficulties are not observed using this mesh.

\section{CONCLUDING REMARKS}

We have briefly reviewed a displacement-pressure $(u / p)$ finite element formulation intended primarily for the analysis of almost semideformable materials, including almost incompressible materials. The examples presented show the use of the $u / p$ formulation in orthotropic analysis and in rubber analysis.

In the orthotropic analysis, the material was chosen so that the pressure was decoupled from the strains, yet the material was not incompressible. The rubber analyses demonstrated the inclusion of contact conditions, the modeling of a simple threedimensional problem and some solution difficulties associated with large pressures.

\section{REFERENCES}

1. M. H. Gadala, Numerical solutions of nonlinear problems of continua-II. Survey of incompressibility constraints and software aspects. Comput. Struct. 22, 841-855 (1986).

2. L. R. Herrmann, Elasticity equations for incompressible and nearly incompressible materials by a variational theorem ALAA J. 3, 1896-1900 (1965).

3. S. W. Key, A variational principle for incompressible and nearly-incompressible anisotropic elasticity. Int. $J$. Solids Struct. 5, 951-964 (1969).

4. M. Bercovier, Y. Hasbani, Y. Gilon and K. J. Bathe, On a finite element procedure for nonlinear incompressible elasticity. In Hybrid and Mixed Finite Element Methods (Edited by S. N. Atluri et al.), Chap. 26.
J. Wiley and Sons, Chichester (1983).

5. B. Häggblad and J. A. Sundberg, Large strain solutions of rubber components. Comput. Struct. 17, 835-843 (1983)

6. A. B. Zdunek and $M$. Bercovier, Numerical evaluation of finite element methods for rubber parts. Proceedings of the Sixth International Conference on Vehicle Structural Mechanics. Society of Automotive Engineers, Detroit (1986).

7. T. Sussman and K. J. Bathe, A finite element formulation for nonlinear incompressible elastic and inelastic analysis. Comput. Struct. 26, 357-409 (1987).

8. K. J. Bathe, Finite Element Procedures in Engineering Analysis. Prentice-Hall, Englewood Cliffs, New Jersey (1982).

9. F. Brezzi, On the existence, uniqueness and approximation of saddle-point problems arising from Lagran gian multipliers. R.A.I.R.O. 8, B-R2, 129-151 (1974).

10. J. T. Oden and N. Kikuchi, Finite element methods for constrained problems in elasticity. Int. J. Numer. Meth. Engng 18, 701-725 (1982).

11. F. Brezzi and K. J. Bathe, Studies of finite element procedures-the Inf-Sup condition, equivalent forms and applications. In Reliability of Methods for Engineering Analysis (Edited by K. J. Bathe and D. R. J. Owen). Pineridge Press, U.K. (1986).

12. ADINA System 5.0 Users Manuals, ADINA R \& D Inc., 71 Elton Ave., Watertown, MA, U.S.A. (1987).

13. P. B. Lindley, Compression characteristics of laterallyunrestrained rubber O-rings. J. Instn Rubb. Ind. 4, 209-213 (1967).

14. P. B. Lindley, Load-compression relationships of rubber units. J. Strain Anal. 1, 190-195 (1966).

15. T. Sussman and K. J. Bathe, Studies of finite element procedures-stress band plots and the evaluation of finite element meshes. Engng Comput. 3, 178-191 (1986).

16. R. S. Rivlin, Large elastic deformations of isotropic materials III. Some simple problems in cylindrical polar co-ordinates. Phil. Trans. A 240, 509-525 (1948). 